\title{
COVID-19 and skin: Analysis of the available data
}

\author{
Martina Yaneva1', Zdravka Demerdjieva', Razvigor Darlenski ${ }^{1,2}$, Nikolay Tsankov ${ }^{1}$ \\ ${ }^{1}$ Department of Dermatology and Venereology, Acibadem City Clinic, Tokuda Hospital, Sofia, Bulgaria, ${ }^{2}$ Department of \\ Dermatology and Venereology, Medical Faculty, Trakia University, Stara Zagora, Bulgaria
}

Corresponding author: Dr. Martina Yaneva, E-mail: yaneva.martina@yahoo.com

\begin{abstract}
Background: This review aims to search the literature for possible cutaneous involvement in patients with COVID-19 infections. Such an analysis could provide dermatologists and other physicians with valuable information on the possible clinical manifestations of the disease as well as suggest a certain immunological pathway of the infectious process. Materials and Methods: A thorough search for the online literature available in scientific databases was conducted. Currently, data on cutaneous involvement is scarce, but, nonetheless, present. The papers found were divided into three categories - reported cases, exacerbations of preexisting skin diseases, and skin manifestations of preventive measures and treatment—and analyzed. Discussion: Dermatologists should take into consideration a COVID-19 infection in patients with diffuse exanthems, whether petechial, vesicular, urticaria-like, or otherwise. Specific patterns of lesions of the skin or of mucous membranes in COVID-19 infections have not yet been defined. Dermatologists should be expecting to deal with aggravated preexisting skin diseases, as well as deal with and minimize skin problems caused by the use of personal protective equipment (PPE).
\end{abstract}

Key words: COVID-19; Cutaneous manifestations; Exacerbation

\section{INTRODUCTION}

In late December 2019, a pneumonia outbreak of unknown etiology emerged in Wuhan, Hubei Province, China, and spread quickly nationwide [1]. On February 11, 2020, the World Health Organization (WHO) designated the new coronavirus disease COVID-19 [2]. By mid-February, COVID-19 has rapidly spread through China and across the world [3], and a pandemic situation was announced on March 11 [4,5].

Coronaviruses (CoVs) are positive-stranded RNA viruses with a crown-like appearance due to the presence of spike glycoproteins on the envelope [6]. Members of the large family of viruses Coronaviridae can cause respiratory, enteric, hepatic, and neurological diseases in various animal species. As with other respiratory pathogens, the transmission of COVID-19 is believed to occur through respiratory droplets from coughing and sneezing. Aerosol transmission is also possible when in protracted exposure to elevated aerosol concentrations in closed spaces [7].

The most common symptoms of the disease are cough, shortness of breath and fever [8].

\section{Reported Cases of COVID-19 Cutaneous Manifestations}

COVID-19 is considered not to be dermatotropic [9], primarily affecting the respiratory system. However, skin manifestations were observed in about one-fifth of a group of patients with COVID-19 in Alessandro Manzoni Hospital, Lecco, northern Italy [10]. 148 COVID-19-positive patients were studied, with 60 of them excluded due to a new medicine intake in the preceding 15 days. Out of the remaining 88 patients, 18 (20.4\%) had developed cutaneous manifestations; 8 had developed skin involvement at the onset, and 10 after hospitalization. Cutaneous manifestations included erythematous rash (14 patients), widespread urticaria (3

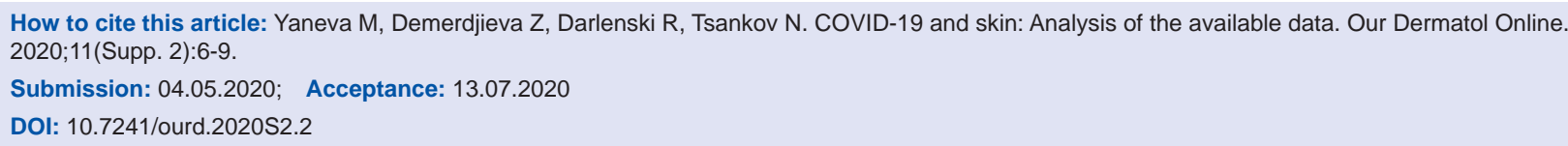


patients), and chickenpox-like vesicles (1 patient). The trunk was the most affected. Itching was low or absent, and lesions usually healed in a few days. No correlation was found with the disease's severity [10].

In all these cases, a possible allergic reaction to any kind of treatment was excluded as most allergic reactions occur within hours to two weeks after taking medication, depending on the mechanism of the cutaneous sensitization [11]. The clinical findings could be interpreted as a nonspecific viral exanthem. A viral exanthem is a widespread nonspecific rash usually characterized by a generalized eruption of erythematous papules and macules $[12,13]$. Certain exanthems have distinct patterns of rashes and prodromal (pre-rash) symptoms, which aid in discriminating the causative virus. In many cases, however, an accurate diagnosis cannot be established on the basis of a clinical examination alone. Medical history may be helpful in evaluating patients, specifically their contact with the disease, immunization record, previous exanthematous illnesses, and associated prodromal symptoms [14,15].

In March 2020, a case of a COVID-19 infection with the presence of a petechial skin rash was reported in Thailand. The patient was initially misdiagnosed with Dengue fever by the first physician in-charge because of the characteristic rash and a low platelet count. In this case, the patient presented further respiratory problems and was referred to a tertiary medical center. Other common virus infections that might cause fever, rash, and respiratory problems were ruled out by a laboratory investigation, and the final diagnosis of a COVID-19 infection was ascertained by a Real-Time PCR (RT-PCR) [16].

Because immune and coagulation changes in viremia can lead to the occlusion of small blood vessels, petechiae, tiny bruises, and transient livedoid eruptions may be present. A case series from Wuhan was published in an NEJM correspondence [17]. In March 2020, Chinese physicians reported the results of a retrospective study that they had conducted. The study included 7 critical COVID-19 patients with acro-ischemia in a single center in Wuhan from February 4 to February 15, 2020. All of the patients had acro-ischemia manifestations, including finger and toe cyanosis, skin bullae, and dry gangrene. Levels of D-dimers, fibrinogens, and fibrinogen degradation products (FDPs) were significantly elevated in most patients. The prothrombin time (PT) was prolonged in 4 patients. D-dimer and FDP levels increased progressively with the exacerbation of COVID-19 infections, and 4 patients were diagnosed with definite disseminated intravascular coagulation (DIC) [18]. However, a differential diagnosis of DIC can be difficult to reach in COVID-19 patients as proinflammatory cytokines and other mediators are capable of activating the coagulation system and downregulating important physiologic anticoagulant pathways [19]. Therefore, the little data available makes it impossible to reach definite conclusions on whether the skin changes described above are due to a general antiviral or critical illness reaction, to an activated autoimmune response, or to a specific COVID-19 mechanism.

Dermatologists should always keep in mind a possible COVID-19 infection in a patient with skin changes accompanied by other symptoms.

Conceivably, many patients with a COVID-19 infection manifesting a rash and experiencing other severe symptoms do not contact a dermatologist, rather physicians of other specialties. Severe symptoms of a life-threatening condition, such as breathing difficulties, chest pain, cyanosis of the lips or face, demand immediate intensive care, and the condition of the skin should rest aside.

\section{Exacerbations of Preexisting Skin Diseases}

Although COVID-19 does not primarily affect the skin, skin changes in the course of a viral infection should not be ignored. Dermatologists should also be expecting aggravations of preexisting chronic and autoimmune skin illnesses, such as atopic dermatitis, psoriasis, seborrheic dermatitis, lupus erythematosus, and hidradenitis suppurativa. Because diseases with an epidermal barrier interruption can facilitate viral acquisition through indirect contact [20], dermatology patients might be at increased risk of developing this infection [9]. Individuals on disease-modifying therapies (DMTs), such as immunomodulatory treatments, are also at higher risk of contracting a COVID-19 infection [21].

Clinical data also suggests that further deterioration in infected patients may be the result of a proinflammatory state created by a cytokine storm [22]. The cytokinestorm immunopathology of SARS-CoV-2 suggests that a subset of immunosuppressive therapies may begin to play a protective role in infected patients. By inhibiting the intensity of the cytokine storm, immunosuppressants may prevent lung tissue damage and further clinical deterioration. There exist, however, 
no universally accepted guidelines. Furthermore, immunomodulators, including biologic agents used to treat cutaneous diseases, may, possibly, predispose to a more severe presentation of a COVID-19 infection. Some recommendations advocate for an immediate discontinuation of all immunomodulators used to treat skin diseases, except corticosteroids [23]. Others, such as the British Dermatology Society, recommend shielding [24]. Dermatologists should employ clinical judgment, existing AAD guidelines, and an understanding of pathophysiology to weigh the risk-benefit ratio of using systemic immunomodulating therapies [25] and the phase of the COVID-19 infection (inflammatory or immune) [26].

An aggravation of preexisting autoimmune or chronic skin diseases with no other signs and symptoms may be the first indication of a concomitant viral infection. Therefore, dermatologists should stay alert to ask their patients about possible previous contact with an infected person or, perhaps, suggest a diagnostic test for COVID-19. It is important to stress the importance of preventive measures, which should be adhered to by both the physician and patient.

\section{Skin Manifestations of Preventive Measures and Treatment}

Skin problems related to personal protective equipment (PPE) and personal hygiene measures are mainly due to the hyperhydration of PPE, friction, epidermal barrier breakdown, and contact reactions, all of which may aggravate existing skin diseases (Figs. 1 and 2) $[9,27]$.

Although no antiviral treatments specific to COVID-19 infections have been approved, several approaches have been proposed, such as lopinavir/ritonavir (400/100 mg every 12 hours), chloroquine (500 mg every 12 hours), and hydroxychloroquine (200 mg every 12 hours). Interferon alfa-e.g., 5 million units by aerosol inhalation twice a day-is also used [7].

The use of lopinavir/ritonavir may sometimes lead to allergic reactions manifested by rash, itching, swelling-especially of the face, tongue, and throatsevere dizziness, and shortness of breath [28]. The side effects of chloroquine include skin itchiness, changes in skin color, hair loss, and skin rashes [29,30]. The most common skin-related side effects of hydroxychloroquine include a bluish-gray pigmentation of the skin, transverse pigmented nail bands, and mucosal pigmentation. Diffuse morbilliform rashes and urticarial and lichenoid eruptions are less common, but

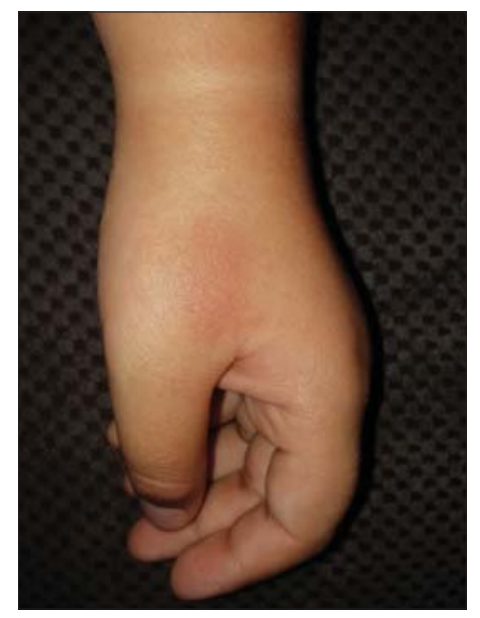

Figure 1: Irritant contact dermatitis in patient 1, a consequence of intensified personal hygiene measures. (Acibadem City Clinic Tokuda Hospital, Sofia, Bulgaria; Image provided by Assoc. Prof. Razvigor Darlenski, M.D., Ph.D.).

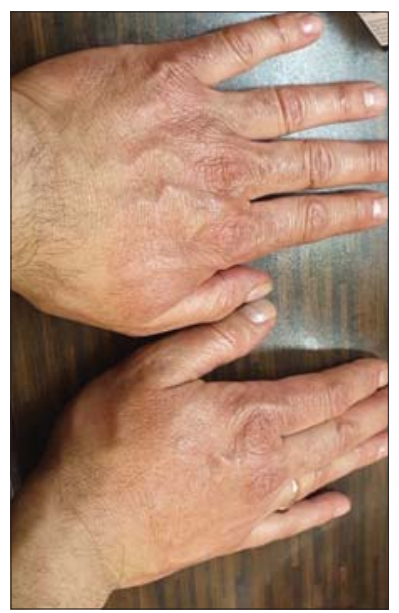

Figure 2: Irritant contact dermatitis in patient 2, a consequence of intensified personal hygiene measures. (Acibadem City Clinic Tokuda Hospital, Sofia, Bulgaria; Image provided by Assoc. Prof. Razvigor Darlenski, M.D., Ph.D.).

can easily be misdiagnosed as a viral-induced eruption instead of a cutaneous side effect. Hydroxychloroquineinduced erythroderma has also been reported in the literature [31]. Synthetic antimalarial drugs (SADs) can deteriorate [32] or even provoke a de novo appearance of psoriasis [33]. Interferon alpha can induce skin rashes, fungal infections, and edema of the extremities.

In extreme cases, irritative or allergic dermatitis can be induced by the contact, local pressure, or maceration of the skin by oxygen supply devices.

\section{CONCLUSION}

Dermatologists should take into consideration a COVID-19 infection in patients with diffuse exanthems, whether petechial, vesicular, urticaria-like, 
or otherwise. Specific patterns of lesions of the skin or of mucous membranes in COVID-19 infections have not yet been defined. Dermatologists should be expecting to deal with aggravated preexisting skin diseases, as well as deal with and minimize skin problems caused by the use of personal protective equipment (PPE).

\section{Consent}

The examination of the patient was conducted according to the Declaration of Helsinki principles.

The authors certify that they have obtained all appropriate patient consent forms. In the form the patient(s) has/have given his/her/ their consent for his/her/their images and other clinical information to be reported in the journal. The patients understand that their names and initials will not be published and due efforts will be made to conceal their identity, but anonymity cannot be guaranteed.

\section{REFERENCES}

1. Xie M, Chen Q. Insight into 2019 novel coronavirus- an updated interim review and lessons from SARS- CoV and MERS- CoV. Int J Infect Dis. 2020, https://doi.org/10.1016/j.ijid.2020.03.071

2. www.who.int/emergencies/diseases/novel-coronavirus-2019/ technical-guidance/naming-the-coronavirus-disease-(covid-2019)and-the-virus-that-causes-it

3. Sanche S, Lin YT, Xu C, Romero-Severson E, Romero-Severson E, Hengartner N, Ke R. High contagiousness and rapid spread of severe acute respiratory syndrome coronavirus 2. Emerg Infect Dis. 2020, https://doi.org/10.3201/eid2607.200282

4. Ng OT, Marimuthu K, Chia PY, Koh V, Chiew CJ, Wang LD, et al. SARS-CoV-2 Infection among travelers returning from Wuhan, China. N Engl J Med. 2020; 382:1476-8.

5. www.who.int/dg/speeches/detail/who-director-general-s-openingremarks-at-the-media-briefing-on-covid-19---11-march-2020

6. Walls Ac, Park Yj, Tortorici Ma, Wall A, McGuire AT, Veesler D. Structure, Function, and Antigenicity of the SARS-CoV-2 Spike Glycoprotein. Cell. 2020; doi: 10.1016/j.cell.2020.02.058.

7. Cascella M, Rajnik M, Cuomo A, Dulebohn SC, Di Napoliet D. Features, Evaluation and Treatment Coronavirus (COVID-19). StatPearls [Internet]. https://www.ncbi.nlm.nih.gov/books/ NBK554776/

8. www.mayoclinic.org/diseases-conditions/coronavirus/symptomscauses/syc-20479963

9. Darlenski R., Tsankov N. Covid-19 pandemic and the skin- What should dermatologists know? Clin Dermatol. 2020, https://doi. org/10.1016/j.clindermatol.2020.03.012

10. Recalcati S. Cutaneous manifestations in COVID-19: a first perspective. JEADV. 2020 doi:10.1111/JDV.16387

11. Ardern-Jones M, Friedmann P. Skin manifestations of drug allergy. Br J Clin Pharmacol. 2011. 71 (5): 672-683.

12. Sarkar R, Mishra K, Garg VK. Fever with rash in a child in India. Indian J Dermatol Venereol Leprol. 2012;78:251-62.

13. Drago F, Ciccarerese G, Gasparini G, et al. Contemporary infectious exanthems: an update. Future Microbiology.2016; 12(2): https:// doi.org/10.2217/fmb-2016-0147

14. Scott LA, Stone MS. Viral exanthems. Dermatol Online J. 2003; 9: 4.

15. Salavastru C, Stanciu A, Fritz K, et al. A burst in the incidence of viral exanthems. Indian Dermatol Online J. 2014; 5(2):144-147.

16. Joob B, Wiwanikit V. COVID- 19 can present with a rash and be mistaken for Dengue, J American Acad Dermatol (2020) https:// doi.org/10.1016/j.jaad.2020.03.036 .

17. www.nejm.org/doi/full/10.1056/NEJMc 2007575? query $=$ featured_coronavirus.

18. Zhang $\mathrm{Y}, \mathrm{Cao} \mathrm{W}, \mathrm{Xiao} \mathrm{M}$ et al. Clinical and coagulation characteristics of 7 patients with critical COVID-19 pneumonia and acro-ischemia. Zhonghua Xue Ye Xue Za Zhi 2020; 41(0): E006.

19. Levi M, Van der Poll T. Inflammation and coagulation. Critical Care Medicine .2010, Vol 38, p S26-S34

20. Tao J, Song Z, Yang L, et al. Emergency management for preventing and controlling nosocomial infection of 2019 novel coronavirus: implications for the dermatology department. Br J Dermatol. 2020.

21. https://pulmonaryfibrosisnews.com/information-about-covid-19for-pulmonary-fibrosis-patients /

22. Huang C, Wang Y, Li X, et al. Clinical features of patients infected with 2019 novel coronavirus in Wuhan, China. Lancet. 2020;395(10223):497-506.

23. Wang Ch, Rademaker M, Baker Ch. et al. COVID-19 and the use of immunomodulatory and biologic agents for severe cutaneous disease: an Australia/New Zealand Consensus Statement. [published online 2020 Apr 7]. Australas J Dermatol. 2020;10.1111/ ajd.13313. doi:10.1111/ajd.13313

24. www.bad.org.uk/shared/get-file.ashx?item type $=$ document\&id $=6674$

25. Shah P, Zampella J. Use of Systemic Immunomodulatory Therapies During the Coronavirus Disease 2019 (COVID-19) Pandemic https://doi.org/10.1016/j.jaad.2020.03.056

26. Siddiqi H, Mehra M. COVID-19 illness in native and immunosuppressed states: a clinical - therapeutic staging proposal. Journal of Heart and Lung Transplantationhttps://doi. org/10.1016/j.healun.2020.03.012

27. Kazandjieva J., Tsankov N., Darlenski R. Aquagenic Syringeal Acrokeratoderma due to extreme hand hygene during the Covid-19 pandemic. Skinmed.2020. in press

28. www.webmd.com/drugs/2/drug-19938-3294/lopinavir-ritonaviroral/lopinavir-ritonavir-solution-oral/details

29. www.drugs.com/sfx/chloroquine-side-effects.html Retrieved 22 March2020.

30. "Chloroquine: MedlinePlus Drug Information". medlineplus.gov. Retrieved 22 March2020.

31. Pai S, Sudershan B, Kuruvilla M, et al. Hydroxychloroquine-induces erythroderma. Indian J Pharmacol. 2017; 49(1): 132-134

32. Tsankov N., Angelova I., Kazandjieva J. Drug induced psoriasis. Recodnition and management. Am J Clin Dermatol, 200; 1(3): 159-165

33. Tsankov N., Stoimenov A., Lazarova A. Psoriasis induit par la Chloroquine chez un malade ayant lupus erithemateux discoid. Rev Sur Dermatol MST, 1990, 2, 453-458

Copyright by Martina Yaneva, et al. This is an open access article distributed under the terms of the Creative Commons Attribution License, which permits unrestricted use, distribution, and reproduction in any medium, provided the original author and source are credited. Source of Support: Nil, Conflict of Interest: None declared. 